\title{
Safe, Affordable, Convenient: Environmental Features of Malls and Other Public Spaces Used by Older Adults for Walking
}

\author{
Diane K. King, Peg Allen, Dina L. Jones, David X. Marquez, \\ David R. Brown, Dori Rosenberg, Sarah Janicek, Laila Allen, and Basia Belza
}

\begin{abstract}
Background: Midlife and older adults use shopping malls for walking, but little research has examined mall characteristics that contribute to their walkability. Methods: We used modified versions of the Centers for Disease Control and Prevention (CDC)-Healthy Aging Research Network (HAN) Environmental Audit and the System for Observing Play and Recreation in Communities (SOPARC) tool to systematically observe 443 walkers in 10 shopping malls. We also observed 87 walkers in 6 community-based nonmall/nongym venues where older adults routinely walked for physical activity. Results: All venues had public transit stops and accessible parking. All malls and 67\% of nonmalls had wayfinding aids, and most venues (81\%) had an established circuitous walking route and clean, well-maintained public restrooms (94\%). All venues had level floor surfaces, and one-half had benches along the walking route. Venues varied in hours of access, programming, tripping hazards, traffic control near entrances, and lighting. Conclusions: Despite diversity in location, size, and purpose, the mall and nonmall venues audited shared numerous environmental features known to promote walking in older adults and few barriers to walking. Future research should consider programmatic features and outreach strategies to expand the use of malls and other suitable public spaces for walking.
\end{abstract}

Keywords: environment, behavior, physical activity, observation, aging

Adults over 50 years of age are the most inactive demographic in the United States despite known health benefits from regular physical activity (PA). ${ }^{1}$ For community-dwelling midlife to older adults, barriers to PA include lack of an exercise companion, lack of access to facilities, and fear of falling, suggesting the importance of social-ecological interventions that promote access to safe venues and support for walking. ${ }^{2,3}$

Studies have identified environmental aspects of neighborhoods that are barriers to walking outdoors for some older adults, including concerns about safety from traffic and crime, limited resting places, tripping hazards, precipitation, and temperature extremes..$^{4-9}$ Sidewalks and streets impose severe challenges for walkers with physical and/or cognitive impairments, due to the increased environmental demands of uneven or slippery surfaces, curbs, crowds, and cars. ${ }^{5,10-13}$ Known facilitators of walking in older adults, such as social support and interesting destinations, may be lacking in many communities. . $^{214,15}$

Mall walking has long been popular with older adults, ${ }^{16,17}$ and malls are frequently recommended by health-care professionals as alternatives to gyms or outdoor walking, particularly for people with neuropathy, arthritis, or sensory or cognitive impairment, because of their presumed safe, low-cost, and pedestrian-friendly features. ${ }^{18,19}$

King (dkking@uaa.alaska.edu) and L Allen are with the Center for Behavioral Health Research and Services, University of Alaska, Anchorage, AK. P Allen is with the George Warren Brown School of Social Work, Washington University, St. Louis, MO. Jones is with the Dept of Orthopaedics and Division of Physical Therapy, West Virginia University, Morgantown, WV. Marquez and Janicek are with the Dept of Kinesiology and Nutrition, University of Illinois at Chicago. Brown is with the Centers for Disease Control and Prevention, Atlanta, GA. Rosenberg is with the Dept of Physical Activity Research, Group Health Research Institute, Seattle, WA. Belza is with the Health Promotion Research Center, University of Washington, Seattle, WA.
The current literature on the use of malls as walking venues for midlife to older adults includes primarily descriptive studies with small sample sizes..$^{20}$ Although there is some recent evidence that shopping mall availability may predict greater physical activity in adults ${ }^{21,22}$ and older adults, ${ }^{23}$ all of these studies categorized walking in the mall as a form of active transportation, which is defined as any self-propelled, human-powered mode of transportation (ie, walking or cycling to reach a destination), rather than a form of exercise whose primary goal is to sustain health and fitness. There is limited research on the environmental and programmatic features of malls where older adults walk for exercise, as well as the characteristics of the walkers themselves. ${ }^{22} \mathrm{We}$ conducted environmental audits at 10 malls and 6 other nonmall/nongym venues (eg, zoo, botanical garden, indoor ice rink, and repurposed factory, described hereafter as nonmalls) and systematically observed the demographic and walking characteristics of 530 walkers. We describe the observed attributes of the walkers and venues to provide a foundation for designing studies that will elucidate the potential influence of these environmental and programmatic variables on older adult walking. Our findings will also be useful to public health and others interested in identifying favorable older adult walking environments within their communities.

\section{Methods}

\section{Mall/Nonmall Selection Criteria}

Investigators in Alaska, Illinois, Missouri, Washington, and West Virginia located 2 to 3 indoor shopping malls in each state that were perceived as popular places for seniors to walk based on advice from local aging services experts, mall website information, and investigator observation. Other criteria included designated walking times outside of regular business hours and potential to reach diverse seniors (ie, urban, suburban, income, population demographics). To explore the potential for safe public walking venues in rural 
and other communities that may lack a suitable (or any) mall, each investigator also identified 1 to 2 public settings (nonmalls) that had established designated times for walking and were similarly identified as places where older adults specifically came to walk for physical activity. All of these settings had a primary business purpose other than physical activity. Fitness facilities and tracks were excluded.

\section{Mall/Nonmall Recruitment Methods}

Twenty-two malls and 7 nonmalls were invited to participate in the study. Venue managers were contacted by phone, e-mail, or in person by a member of the research team and provided written information about the purpose of the study and a description of data collection procedures.

\section{Preparation for Data Collection}

Peak walking times for older adults were identified by venue managers and verified, where possible, by visits to the venues before formal data collection. Similarly, through venue managers, observations, and discussions with walkers during designated hours, common walking routes (ie, "circuits") were determined.

\section{Measures}

We used the previously validated System for Observing Play and Recreation in Communities (SOPARC), ${ }^{24}$ an instrument that uses momentary time sampling methods to quantify the number and activity levels of people using a defined space for PA during a specified observation period. SOPARC allows for comparison of observed activity levels of different demographic groups and for calculation of a total energy expenditure rate among all observed walkers that can be used to compare diverse venues. Observers sat or stood in a predetermined location along the walking route, using a paper coding form to tally each walker's gender, age group, race/ ethnicity, and activity level as soon as they crossed an imaginary "screen" line in front of the observer. Descriptive categories were based soley on the observers' estimation of the individual's gender (male or female), age (child appearing to be 12 years or under, teen appearing to be 13-19 years, adult appearing to be 20-64 years, or senior appearing to be 65 years or older), and race/ethnicity (white or nonwhite). ${ }^{24}$ Activity level categories were sedentary, which was defined as lying down, sitting, or standing in place (a seldom used code while observing a walking circuit); walking, which was defined as walking at a casual pace; and vigorous, which was defined as any activity requiring more effort than casually paced walking (eg, brisk-paced walking, walking with ankle or wrist weights). Observers also noted use of a mobility aide such as a cane, walker, shopping cart, scooter, or wheelchair.

One count was conducted at each venue during the preidentified peak older adult walking times for the estimated period of time it would take for an older adult to complete 1 circuit at a casual pace of $2.5 \mathrm{mph}$, considered an average speed for adults 65 years and older. ${ }^{25}$ Before starting the observation period, trained observers measured the length of each walking circuit in feet via a Rolatape (RT13) 12-inch measuring wheel, converted feet to miles, and divided route length by $2.5 \mathrm{mph}$ to estimate the appropriate observation time.

Our 70-item environmental audit instrument was an adapted version of the CDC-Healthy Aging Research Network (HAN) environmental audit tool. ${ }^{2,26}$ Features observed included the external environment (eg, public transit, parking, entrances, physical and social order), internal environment (eg, wayfinding aids, amenities, physical and social order), and walking environment (eg, hours, resources, routes, conditions) (see Online Appendix A).

Local research teams performed venue audits using a written guide adapted from user guides for SOPARC ${ }^{24}$ and the HAN environmental audit tool. ${ }^{26,27}$ We developed the guide to enhance fidelity of data collection procedures and piloted and refined it, along with the coding forms, before data collection. We established interrater consistency at venues by having pairs of auditors complete initial observations separately, discuss coding discrepancies, and reach consensus. All protocols were approved by the Institutional Review Boards of the University of Washington and 3 of the additional participating universities. Auditors entered data into a central, secure database that was aggregated and analyzed by University of Washington.

We used descriptive statistics to summarize venue participation rates, mall and nonmall environmental characteristics (by audit domains of external, internal, and walking environments), and walker demographic characteristics (from SOPARC data), as appropriate. To compare observed environmental features and characteristics of observed mall walkers by malls versus nonmalls, we conducted $t$ tests, $\chi^{2}$ tests, and, where needed because of small cell sizes, Fisher's exact tests. Analyses were conducted using MS Excel (Microsoft, Redmond, WA) and SPSS, version 22 (IBM, Armonk, NY).

To compare use and intensity of PA occurring across venues, we used a validated SOPARC formula to calculate estimated energy expenditure (EE) as the number of people counted in the sedentary, walking, and vigorous categories, multiplied by the constants 0.051 $\mathrm{kcal} / \mathrm{kg} / \mathrm{min}, 0.096 \mathrm{kcal} / \mathrm{kg} / \mathrm{min}$, and $0.144 \mathrm{kcal} / \mathrm{kg} / \mathrm{min}$, respectively, then summed to provide a value that can be interpreted as the number of kilocalories per kilogram of body weight per minute expended in each venue during the observation period. ${ }^{28}$ These EE rates are dependent on the number of people observed who are engaged in varying intensity levels of PA during the observation window. To compare EE rates between the malls and nonmalls, EE for each venue type were summed and divided by number of venues observed in each category. ${ }^{24}$

\section{Results}

\section{Characteristics of Walking Venues}

Ten of the $22(46 \%)$ invited malls and 6 of the 7 (86\%) invited nonmalls agreed to participate in the study. Participating venues were situated within geographically diverse communities that varied by population density, demographics, and climate. Of the 10 malls, 8 were in suburban or urban parts of large metropolitan statistical areas, and 2 were in small cities. Two malls were situated within lower-income areas, 6 were in middle-income areas, and 2 were in higher-income areas. Five malls had more than 1 million square feet, and the smallest had 143000 square feet (median 993979 square feet). All malls were primarily retail establishments containing from 50 to 200 stores, with a median of 120 stores. All but 2 were built between 1974 and 1983. Five malls had at least some stores with discounted pricing, 4 had moderately priced stores, and 1 was an upscale mall. All malls were open daily for walking before store business hours, but varied with regard to amount of time allotted for walking before businesses were open (ie, 1.5-4.5 hours), length of walking route (ie, 954-4170 feet), walking program structure, and walker amenities.

Nonmalls were business, attraction, or recreation destinations and included 2 indoor ice rinks, an outdoor botanical garden, an 
outdoor zoo, a former mall (now used by a variety of nonretail nonprofits and businesses), and a former factory (converted to a multipurpose community facility). Walking hours at nonmalls were highly variable, ranging from sunrise to sunset to limited times and days that varied by season. The length of walking routes also varied considerably at nonmalls, ranging from a 728-foot loop at an ice skating rink to a 13,576-foot meandering path through a botanic garden.

Nonparticipating malls and nonmalls were similar to participating venues in location and population demographics. Nonparticipating malls were primarily in urban or suburban settings $(n=11)$, with 2 in small cities. Two of the nonparticipating malls advertised as upscale retail and were located in high income communities, 6 were in middle income areas, and 4 were in lower income communities. The nonparticipating nonmall was a museum with a walking program that was located in the center of a large city. Reasons for nonparticipation were lack of interest $(n=6)$, company rules or legal barriers to research participation $(n=3)$, no response to invitations $(\mathrm{n}=2)$, and agreed after recruitment targets were met $(\mathrm{n}=2)$.

\section{Environmental Audit Results}

Table 1 summarizes and compares the mall and nonmall observed characteristics. For all venue types, external environments had minimal trip/fall hazards. All locations had adequate parking, either on-site or in a nearby garage. Parking lots were generally rated as "well-lit with good visibility for both motorists and pedestrians," with the exception of 1 mall parking lot that was rated as "dimly lit" and 1 nonmall lot rated as "dark and dangerous." Designated, marked walkways and traffic control were lacking in most of the mall parking lots. Indoor environments at all malls and nonmalls included aesthetics such as artwork; plants and interesting architecture; presence of "wayfinding" aids (ie, maps or navigational signage to orient users); benches; and accessible, clean, maintained restrooms. Lighting within 33\% of nonmall restrooms was poor, and $50 \%$ of the malls lacked handrails on stairs and working drinking fountains. Physical disorder such as litter, boarded windows, and broken glass was noted within $31 \%$ of the venues. Social disorder, such as people loitering or engaged in illicit activity, was not observed.

In addition to designated walking hours, a variety of walking support features and resources were observed in mall and nonmall venues, including marked walking routes (malls 50\%, nonmalls $83 \%$ ), walking program leaders (malls $40 \%$, nonmalls $67 \%$ ), as well as program information tables and lockers for walkers. One-half $(50 \%)$ of the nonmalls and $40 \%$ of the malls had structured program features such as posted walking protocols and motivational signs, exercise stations, and health information. Potentially discouraging internal environment features at the malls included physical disorder, such as shuttered stores, bathroom graffiti, and outdated or illegible wayfinding aids. A few trip hazards were observed in malls and nonmalls along walking routes, primarily due to cracked or frayed flooring or mixed flooring materials (eg, transitioning from carpet to tile).

\section{SOPARC Results}

Demographic characteristics of observed mall walkers are summarized in Table 2. A total of 530 walkers were observed in the 16 settings. The majority of walkers were observed as female (57\%), white (78\%), and 65 years or older (63\%). Most walked at a moderate pace (83\%). Walkers at nonmalls were observed as less diverse racially $(P=.003)$ and younger $(P<.001)$ than walkers at the malls. The average EE for the mall locations was nearly 3-fold (mean $=4.4, \mathrm{SD}=3.2$ ) the average $\mathrm{EE}$ at nonmalls (mean $=1.5$, $\mathrm{SD}=0.9$ ). This difference was due primarily to 3 times as many walkers observed, on average, at the mall venues, and was not statistically significant.

\section{Discussion}

Malls are frequently recommended as safe places for older adults to walk, yet research evaluating these venues as components of the built environment that support physical activity is limited. This study reported our use of systematic observational methods to evaluate the walkability features of malls and other nongym venues that are routinely used by older adults to walk for exercise. By assessing the malls on objective environmental variables that have been demonstrated as either facilitators or barriers to older adults when walking in neighborhoods, or on trails, our findings may be used to identify malls and other community spaces that could be used to promote walking in this sedentary and rapidly growing demographic group.

Despite differences in venue size and layout, environmental audit variables were surprisingly consistent between malls and nonmalls, particularly with regard to built environment factors that are associated with older adult walking, including safety from traffic and crime, maintained and even walking surfaces with limited tripping hazards, benches along the walking route, and clean public restrooms. ${ }^{4-15}$ Although a few of the venues provided outdoor walking opportunities, most of the malls and nonmalls were indoors, eliminating climatic barriers such as heat, cold, wind, darkness, and precipitation.

Our systematic observations of the walkers themselves suggest that participants of mall and nonmall walking programs predominantly spend their time walking at a moderate intensity. This was consistent across the 2 types of settings with few individuals being sedentary or engaging in more vigorous walking.

Comparing the characteristics of the walkers in the malls with the walkers in the nonmall venues provided a snapshot of potential differences that could be explored in future research. The total numbers of observed walkers and their demographic characteristics showed some overall variation between malls and nonmall venues, with the malls seeming to attract more older adults, ethnic minorities, and males and the nonmalls attracting a higher proportion of adolescents and adults. Although the reasons for these differences are not known, we observed that several of the nonmalls were less proximal to residential neighborhoods. Burdensome travel time may not be viewed as an accessible option to many older adults, ${ }^{29}$ and proximity to destinations has been demonstrated in other studies as important to regular use. ${ }^{22}$ For example, the botanical garden was 30 miles from the city center. We also observed that nonmalls, rather than having set morning walking hours, 6 to 7 days per week, offered greater variability in designated walking hours. Although this may be a plus for some individuals, it may also deter those older adults preferring a daily year-round option. For example, both of the ice rinks' walking hours varied by day of the week and season, and access to the outdoor venues were subject to weather conditions, such as snow and ice. Another potential barrier with some nonmall venues is that there may be an entry fee associated with use of the setting for walking. Such a fee may limit use by walkers of low income or on a fixed income. This may also be true of mall locations that offer formal exercise classes or other programming, however there were no fees associated with walking at the 10 malls observed for this study. 
Table 1 Observed Environmental Features from Mall Environmental Audit

\begin{tabular}{|c|c|c|c|c|}
\hline \multirow[b]{2}{*}{ Audit domains } & \multirow[b]{2}{*}{ Environment features } & \multicolumn{3}{|c|}{ Audit results } \\
\hline & & Malls $(n=10)$ & Nonmalls $(n=6)$ & $P$ value ${ }^{a}$ \\
\hline \multirow[t]{16}{*}{ External environment } & Public transit stops & $100 \%$ & $67 \%$ & .12 \\
\hline & Sheltered & $50 \%$ & $33 \%$ & .72 \\
\hline & Seating & $60 \%$ & $17 \%$ & .28 \\
\hline & Trip/fall hazards & $40 \%$ & $17 \%$ & .54 \\
\hline & Marked walkway to entrance & $50 \%$ & $17 \%$ & .86 \\
\hline & Distance in feet to entry, average (SD) & $220(230)$ & $303(206)$ & .54 \\
\hline & Parking available on site & $80 \%$ & $83 \%$ & 64 \\
\hline & Close to entry & $100 \%$ & $100 \%$ & 1.0 \\
\hline & Accessible & $90 \%$ & $83 \%$ & .62 \\
\hline & Well lit & $70 \%$ & $67 \%$ & .64 \\
\hline & Marked walkway to entrance & $20 \%$ & $50 \%$ & .86 \\
\hline & Traffic control near walkways & $40 \%$ & $67 \%$ & .61 \\
\hline & Number of entries unlocked, average (SD) & $5(2.62)$ & $2(.75)$ & .004 \\
\hline & Places to rest near entrance & $50 \%$ & $50 \%$ & 1.0 \\
\hline & Aesthetically pleasing & $30 \%$ & $67 \%$ & .30 \\
\hline & Physical disorder ${ }^{\mathrm{b}}$ & $40 \%$ & $0 \%$ & .23 \\
\hline \multirow[t]{14}{*}{ Internal general } & Wayfinding aids & $80 \%$ & $67 \%$ & 60 \\
\hline & Directional signs to key landmarks & $80 \%$ & $67 \%$ & 60 \\
\hline & Information kiosks & $90 \%$ & $83 \%$ & 1.0 \\
\hline & Maps indicating "you are here" & $100 \%$ & $33 \%$ & .008 \\
\hline & Amenities & $100 \%$ & $100 \%$ & 1.0 \\
\hline & Benches & $100 \%$ & $83 \%$ & .38 \\
\hline & Drinking fountains (working) & $50 \%$ & $83 \%$ & .31 \\
\hline & Handrails on stairs & $50 \%$ & $50 \%$ & 1.0 \\
\hline & Payphones & $20 \%$ & $0 \%$ & .50 \\
\hline & Restrooms available & $100 \%$ & $100 \%$ & 1.0 \\
\hline & Clearly marked & $100 \%$ & $67 \%$ & .12 \\
\hline & Well lit & $90 \%$ & $67 \%$ & .52 \\
\hline & Clean/maintained & $90 \%$ & $100 \%$ & 1.0 \\
\hline & Physical disorder ${ }^{\mathrm{c}}$ & $30 \%$ & $33 \%$ & 1.0 \\
\hline \multirow[t]{13}{*}{ Walking environment } & Doors unlocked during nonbusiness hours for walkers & $100 \%$ & $50 \%$ & .04 \\
\hline & Designated walking route & $50 \%$ & $83 \%$ & .31 \\
\hline & Circuit & $90 \%$ & $80 \%$ & 1.0 \\
\hline & Route length in feet, average (SD) & $3365(1970)$ & $3835(5536)$ & .86 \\
\hline & Trip/fall hazards & $30 \%$ & $50 \%$ & 61 \\
\hline & Lighting issues along route & $50 \%$ & $17 \%$ & .31 \\
\hline & Walking support and resources & & & \\
\hline & Structured program $^{\mathrm{d}}$ & $40 \%$ & $50 \%$ & 1.0 \\
\hline & Program leader & $40 \%$ & $67 \%$ & 61 \\
\hline & Other walkers & $90 \%$ & $100 \%$ & 1.0 \\
\hline & Building security visible & $70 \%$ & $17 \%$ & .12 \\
\hline & Welcoming & $60 \%$ & $67 \%$ & 1.0 \\
\hline & No competing use & $50 \%$ & $50 \%$ & 1.0 \\
\hline
\end{tabular}

a $P$ value from 2-sided Fisher's exact test (some cells were too small for $\mathrm{c}^{2}$ test) or $t$ test.

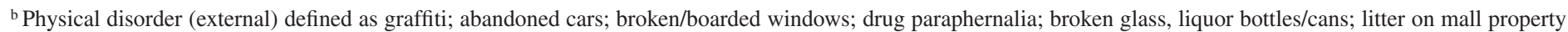
or walkways.

c Physical disorder (internal) defined as graffiti, stores shuttered, debris or overflowing trash cans.

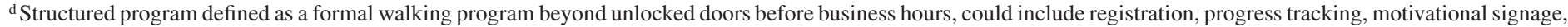
exercise stations and accommodations for walkers of varying ability. 
Table 2 Characteristics of People Observed Walking in 10 Malls and 6 Nonmalls

\begin{tabular}{|c|c|c|c|c|}
\hline \multirow[b]{2}{*}{ Characteristic } & \multirow[b]{2}{*}{ Total $(\mathrm{N}=530)$} & \multicolumn{2}{|c|}{ Venue } & \multirow[b]{2}{*}{$\boldsymbol{P}$ value $^{\mathrm{a}}$} \\
\hline & & Malls $(n=443)$ & Nonmalls $(n=87)$ & \\
\hline \multicolumn{5}{|l|}{ Demographics $^{\mathrm{b}}$} \\
\hline \multicolumn{5}{|l|}{$\operatorname{Sex}(\%)$} \\
\hline Female & 57 & 55 & 67 & .13 \\
\hline \multicolumn{5}{|l|}{ Race/ethnicity (\%) } \\
\hline Nonwhite and/or ethnic minority & 22 & 24 & 8 & .003 \\
\hline Age group (\%) & & & & $<.001$ \\
\hline Children & 0.6 & 0.7 & 0 & \\
\hline Adolescents & 3 & 0.7 & 15 & \\
\hline Adults & 33 & 29 & 54 & \\
\hline Older adults & 63 & 69 & 31 & \\
\hline \multicolumn{5}{|l|}{ Use of assistive device (\%) } \\
\hline $\begin{array}{l}\text { Cane, walker, shopping cart, scooter, } \\
\text { or wheelchair }\end{array}$ & 2.3 & 2.6 & 4.6 & .26 \\
\hline Physical activity level (\%) & & & & .07 \\
\hline Sedentary ${ }^{\mathrm{c}}$ & 4 & 5 & 0 & \\
\hline Walking (moderate) ${ }^{\mathrm{d}}$ & 84 & 84 & 84 & \\
\hline Vigorous $^{\mathrm{e}}$ & 12 & 11 & 16 & \\
\hline Average EE $(S D)^{f}$ & $3.3(2.6)$ & $4.4(3.2)$ & $1.5(0.9)$ & .12 \\
\hline Average observation time in $\min (\mathrm{SD})$ & $20.4(14.3)$ & $20.2(9.2)$ & $20.8(21.4)$ & .93 \\
\hline
\end{tabular}

Abbreviation: EE, energy expenditure rate.

${ }^{a} c^{2}$ tests or $t$ tests.

${ }^{\mathrm{b}}$ Percentages were rounded, where possible, to nearest whole number and were based on walkers who were counted after crossing an imaginary sight line. Observation times at each venue ranged from 3 to $30 \mathrm{~min}$, and were based on the length of time it would take 1 person to complete 1 circuit at a pace of $2.5 \mathrm{mph}$.

c $\%$ persons observed on the walking route who were lying down, sitting, or standing in place.

d \% Persons observed walking at a casual pace.

e \% Persons observed walking at greater than a leisurely pace (eg, brisk walking, race walking, walking with ankle or wrist weights).

${ }^{\mathrm{f}} \mathrm{EE}$ is the total estimated $\mathrm{kcal} / \mathrm{kg}$ expended by all walkers observed in the sedentary, walking, and vigorous categories, multiplied by the constants $0.051 \mathrm{kcal} / \mathrm{kg} / \mathrm{min}$, $0.096 \mathrm{kcal} / \mathrm{kg} / \mathrm{min}$, and $0.144 \mathrm{kcal} / \mathrm{kg} / \mathrm{min}$, respectively. The average EE rates are the sum of the venue-level EE rates divided by the number of venues (ie, average EE for all venues $=53 / 16$ venues; average mall $\mathrm{EE}=44 / 10$ malls; average nonmall $\mathrm{EE}=9 / 6$ nonmall sites).

Our study had several limitations. We used a convenience sample of venues that were located in 5 geographically diverse states. Venues were purposely selected to maximize variety in size, program structure, and community demographics, but do not represent all types of malls and nonmall settings nationally that are used by older adults for walking. Second, although our environmental audit and walker observation tools were informed by well-validated instruments and reviewed by content experts to promote their validity for our target population, they were not validated for research purposes. Resource constraints prohibited centralized training or use of a trained, traveling audit team to assure interobserver consistency and calibration among our 5 states. We attempted to reduce observer bias by creating a written guide and forms and reaching consensus to resolve interpretation discrepancies across states and between raters, but interrater reliability was not formally assessed. Third, the SOPARC counts of mall walkers were conducted during predetermined peak walking hours for older adults, but were limited to 1 week day count per venue due to resource constraints that prohibited multiple weekday and weekend visits to each venue, as recommended ${ }^{24}$; our samples of observed walkers, therefore, do not represent weekly volume or demographics of all walkers. In addition, our calculation of an estimated EE rate for each location was based on a single brief observation period of walkers at each venue. Increasing the number of observations per venue would have provided a better indicator of the typical EE rate for each location. Repeating the observations over time would also be useful to measure the impact of any changes made by the malls, such as promotional efforts to increase walking program reach, or programmatic changes, such as providing walkers with weights or exercise stations that would increase the intensity of their physical activity. Fourth, a surprising finding was the number of mall managers who declined participation in the study despite unlocking their doors early for walkers. It is unknown if the built environment of nonparticipating malls is different from the participating malls or if their walkers differed demographically, despite their representing similar geographic and demographic locations to the participating malls. The large number of refusals may reflect business pressures and desire to limit distractions from commercial priorities, in addition to the stated concerns from a few of the malls regarding participating in an observational research study that included an environmental audit. ${ }^{30}$ 
Strengths of this study include our use of systematic methods to identify consistent features across geographically diverse malls that contribute to their desirability as walking venues for older adults. We also provide new information about nonmall venues that share similar strengths and could potentially be repurposed to increase the number and diversity of safe walking venues in communities without malls.

\section{Conclusions}

Malls and other public venues used by older adults for walking were characterized by the presence of numerous features that promote safe walking environments for seniors and few barriers. Further investigation of strategies to promote their reach and effectiveness is recommended. Research on mall-walking promotional practices and comparative effectiveness of policy, environment, and programmatic approaches and their interactions will help to further our understanding of the potential of malls and other public venues to promote older adult walking.

\section{Acknowledgments}

The authors acknowledge Rebecca Hunter and Thom McKenzie for guidance with the audit; Michael Kelly, Jeremy Thurston, Rebecca Tiffany, and Marc Cormier for assistance with data collection; Laura Farren and Christina E. Miyawaki for administrative support; and study participants. This publication is a product of the University of Washington Health Promotion Research Center, a Centers for Disease Control and Prevention (CDC) Prevention Research Center, and was supported by Cooperative Agreement Number U48-DP001911 and Special Interest Project (SIP) 13-070 from the CDC. The findings and conclusions in this publication are those of the author(s) and do not necessarily represent CDC's official position.

\section{References}

1. King AC, King DK. Physical activity for an aging population. Public Health Rev. 2010;32(2):401-426.

2. Hunter RH, Anderson LA, Belza B, et al. Environments for healthy aging: linking prevention research and public health practice. Prev Chronic Dis. 2013;10:E55. PubMed 10.5888/pcd10.120244

3. Satariano WA, Haight TJ, Tager IB. Reasons given by older people for limitation or avoidance of leisure time physical activity. J Am Geriatr Soc. 2000;48(5):505-512. PubMed doi:10.1111/j.1532-5415.2000. tb04996.x

4. Gallagher NA, Gretebeck KA, Robinson JC, Torres ER, Murphy SL, Martyn KK. Neighborhood factors relevant for walking in older, urban, African American adults. J Aging Phys Act. 2010;18(1):99-115. PubMed

5. King D. Neighborhood and individual factors in activity in older adults: results from the neighborhood and senior health study. J Aging Phys Act. 2008;16:144-170. PubMed

6. Michael YL, Green MK, Farquhar SA. Neighborhood design and active aging. Health Place. 2006;12(4):734-740. PubMed doi:10.1016/j. healthplace.2005.08.002

7. Rosenberg DE, Huang DL, Simonovich SD, Belza B. Outdoor built environment barriers and facilitators to activity among midlife and older adults with mobility disabilities. Gerontologist. 2013;53(2):268279. PubMed doi:10.1093/geront/gns119

8. Sawchuk CN, Russo JE, Bogart A, et al. Barriers and facilitators to walking and physical activity among American Indian elders. Prev Chronic Dis. 2011;8(3):A63. PubMed

9. Yen IH, Flood JF, Thompson H, Anderson LA, Wong G. How design of places promotes or inhibits mobility of older adults: realist synthesis of
20 years of research. J Aging Health. 2014;26(8):1340-1372. PubMed doi: $10.1177 / 0898264314527610$

10. Kerr J, Rosenberg DE, Nathan A, et al. Applying the ecological model of behavior change to a physical activity trial in retirement communities: description of the study protocol. Contemp Clin Trials. 2012;33:1180-1188. PubMed doi:10.1016/j.cct.2012.08.005

11. Prohaska TR, Eisenstein AR, Satariano WA, et al. Walking and the preservation of cognitive function in older populations. Gerontologist. 2009;49(Suppl 1):S86-S93. PubMed doi:10.1093/geront/gnp079

12. Satariano WA, Ivey SL, Kurtovich M, et al. Lower-body function, neighborhoods, and walking in an older population. Am J Prev Med. 2010;38:419-428. PubMed doi:10.1016/j.amepre.2009.12.031

13. Shumway-Cook A, Patla AE, Stewart A, Ferrucci L, Ciol MA, Guralnik JM. Environmental demands associated with community mobility in older adults with and without mobility disabilities. Phys Ther. 2002;82:670-681. PubMed

14. Belza B, Walwick J, Shiu-Thornton S, Schwartz S, Taylor M, Logerfo J. Older adult perspectives on physical activity and exercise: voices from multiple cultures. Prev Chronic Dis. 2004;1(4):A09. PubMed

15. Rosenberg DE, Kerr J, Sallis JF, Norman GJ, Calfas K, Patrick K. Promoting walking among older adults living in retirement communities. J Aging Phys Act. 2012;20(3):379-394. PubMed

16. Duncan HH, Travis SS, McAuley WJ. An emergent theoretical model for interventions encouraging physical activity (mall walking) among older adults. J Appl Gerontol. 1995;14(1):64-77. doi:10.1177/073346489501400105

17. Zenk SN, Wilbur J, Wang E, et al. Neighborhood environment and adherence to a walking intervention in African American women. Health Educ Behav. 2009;36(1):167-181. PubMed doi:10.1177/1090198108321249

18. Cuaderes E, Lamb WL, Alger A. The older adult with diabetes: peripheral neuropathy and walking for health. Nurs Clin North Am. 2014;49(2):171-181. PubMed doi:10.1016/j.cnur.2014.02.005

19. Eyler AA, Brownson RC, Bacak SJ, Housemann RA. The epidemiology of walking for physical activity in the United States. Med Sci Sports Exerc. 2003;35(9):1529-1536. PubMed doi:10.1249/01. MSS.0000084622.39122.0C

20. Farren L, Belza B, Allen P, et al. Mall walking program environments, features, and participants: a scoping review. Prev Chronic Dis. 2015;12:150027. doi: $10.5888 /$ pcd12.150027

21. Chrisman M, Nothwehr F, Janz K, Yang J, Oleson J. Perceived resources and environmental correlates of domain-specific physical activity in rural midwestern adults. J Phys Act Health. 2015;12(7):962967.. PubMed doi:10.1123/jpah.2013-0453

22. McCormack GR, Giles-Corti B, Bulsara M. The relationship between destination proximity, destination mix and physical activity behaviors. Prev Med. 2008;46(1):33-40. PubMed doi:10.1016/j. ypmed.2007.01.013

23. Winters M, Voss C, Ashe MC, Gutteridge K, McKay H, SimsGould J. Where do they go and how do they get there? Older adults' travel behaviour in a highly walkable environment. Soc Sci Med. 2015;133:304-312. PubMed doi:10.1016/j.socscimed.2014.07.006

24. McKenzie TL, Cohen DA. SOPARC (System for Observing Play and Recreation in Communities). Description and procedures manual. http://www.activelivingresearch.org/files/SOPARC_Protocols.pdf. Accessed November 20, 2013.

25. Dumbaugh E. Designing communities to enhance the safety and mobility of older adults: a universal approach. J Plann Lit. 2008;23:17-36. doi: $10.1177 / 0885412208318559$

26. Health Promotion Research Center. Healthy Aging Research Network archives 2001-2004. http://www.prc-han.org/tools-environment. 2013.

27. Kealey M, Kruger J, Hunter R, et al. Engaging older adults to be more active where they live: audit tool development [abstract]. Prev Chronic Dis. 2005;2(2).

28 McKenzie TL, Cohen DA, Sehgal A, Williamson S, Golinelli D. System for Observing Play and Recreation in Communities (SOPARC): reliability and feasibility measures. J Phys Act Health. 2006;3(Suppl 1):S208-S222. PubMed 
29. Richard L, Gauvin L, Kestens Y, et al. Neighborhood resources and social participation among older adults: results from the VoisiNuage Study. J Aging Health. 2013;25(2):296-318. PubMed doi: $10.1177 / 0898264312468487$
30. Peterson H. America's shopping malls are dying a slow, ugly death. Business Insider. 2014. http://www.businessinsider.com/shoppingmalls-are-going-extinct-2014-1. Accessed February 1, 2015. 